\title{
Square core jacketed air-clad fiber
}

\author{
J.R. Hayes, Joanne C. Flanagan, Tanya M. Monro and D.J. Richardson \\ Optoelectronics Research Centre University of Southampton, SO17 1BJ, UK. \\ Please note that Tanya M. Monro is now based at the School of Chemistry and Physics, University of Adelaide, \\ Australia \\ jrh@orc.soton.ac.uk

\section{P. Grunewald and R. Allott} \\ Exitech Ltd. Oxford, OX5 1QU, UK
}

\begin{abstract}
We report the fabrication of a highly multi-mode square core jacketed air-clad fiber with a top-hat near-field intensity profile. Using this fiber to deliver Q-switched pulses at $1 \mu \mathrm{m}$ we have successfully produced square shaped ablation marks in indium tin oxide (ITO) without the use of complex beam shaping optics.
\end{abstract}

(C) 2006 Optical Society of America

OCIS codes: (060.2280) Fiber design and fabrication; (060.2400) Fiber properties

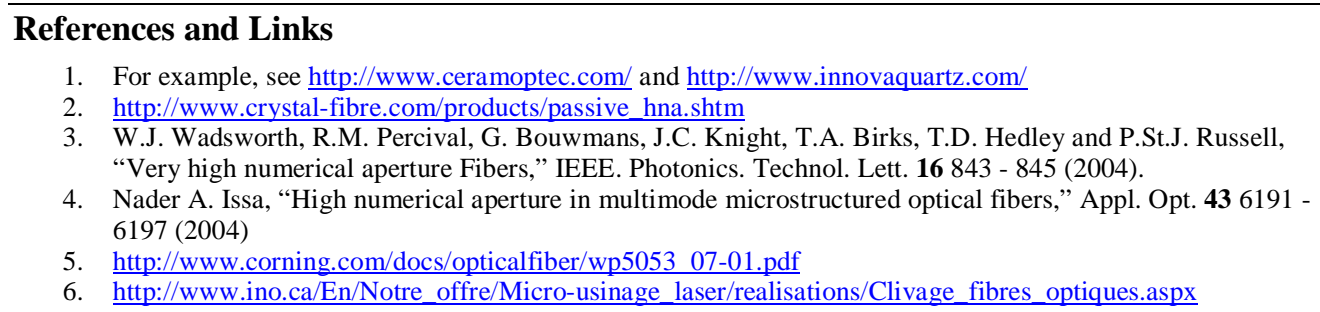

\section{Introduction}

The ideal beam delivery system for an industrial processing tool should supply laser light with the desired shape and intensity profile directly to the work piece, with minimal energy losses. For high power applications, safety considerations require the enclosure of the entire light path, a necessity that introduces design restraints and can also add undesirable bulk and clutter to the manufacturing area. Cabled optical fiber is an attractive delivery option in these instances, offering a safe, compact and flexible solution that also permits bulky sources and their associated pump and cooling systems to be housed remotely from the work area. Using optical fiber delivery also enables light from several separate sources to be transported simultaneously to the same work piece location. As a direct result of these advantages, fiber delivery of intense laser radiation is currently utilized in a wide range of application sectors, from medicine and defense through to the industrial machining and processing of materials. In many of these applications, multi-mode beams with 'top-hat' intensity profiles represent the processing beam of choice. Commercially available large-core fibers, created by cladding a pure silica core with a low-index glass or polymer, are capable of meeting these requirements, but are restricted in application by the limited range of achievable numerical apertures [1].

Silica microstructured fiber technology, in which light is guided via the inclusion of microscopic air tubes, has been shown to offer a practical route towards highly multi-mode, large-core fiber designs [2,3]. An example of this fiber type, shown in cross-section in Fig. 1(a), illustrates how a single ring of air tubes can be used to optically isolate a large fiber core and create a waveguide from a single material. These structures, which are typically referred to as jacketed air-clad (JAC) fibers, not only offer potential power handling advantages over polymer clad designs, since they can be made entirely from pure silica, but also enable a much 
wider range of numerical apertures to be created [3]. The average refractive index of the microstructured region is determined by the width of the supporting struts (shown in higher magnification in Fig. 1(b)) and can be accurately tailored from values approaching those of both pure silica and air simply by changing the strut width [3]. This enables JAC fibers with arbitrarily low values of numerical aperture up to values as a high as 0.9 to be created $[3,4]$, which offers great promise for highly multi-mode beam delivery.

For many applications the delivery of a circular beam is only a partial solution. For example, in certain high power processing applications, such as the surface patterning of thin conductive films for the production of flat panel displays, a square beam shape is highly desirable. In order to achieve this, conventional techniques utilize complex beam shaping processes (that introduce significant losses) to convert the output from delivery fibers/laser systems with circular beams. One obvious way to bypass this additional beam shaping stage is to use a delivery fiber that itself has the desired beam profile. Polymer clad fibers have recently been produced with square core shapes (shown in Fig. 1(c)) and this technology offers great flexibility in terms of core shape. In addition to the numerical aperture restrictions of polymer clad fibers, structural changes take place during fiber drawing where the outer shape of the preform (and hence the core) inevitably experiences a degree of rounding (as illustrated in Fig. 1(c)) due to the surface tension of the softened glass. We note that it may be possible to avoid this rounding for a shaped core held within a circular glass cladding.
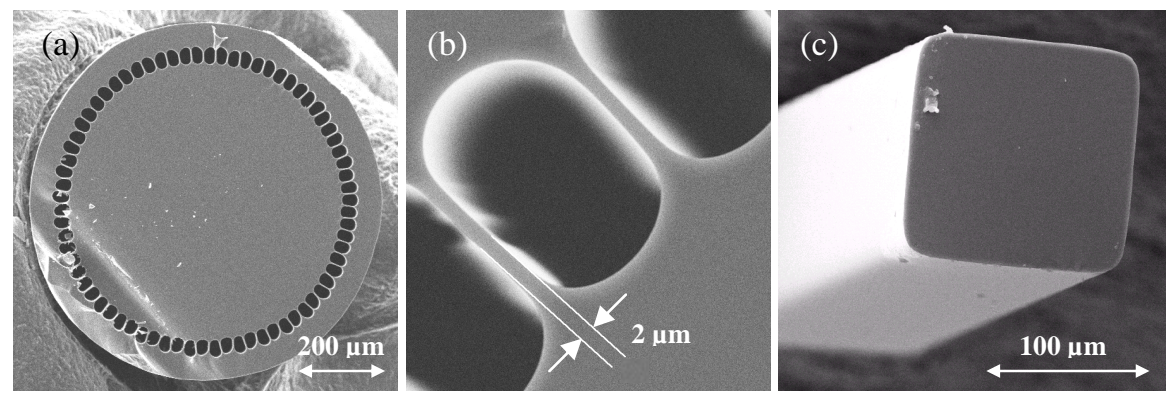

Fig. 1. Scanning electron microscope (SEM) image of a JAC fiber fabricated at the ORC with a core $>600 \mu \mathrm{m}$ in diameter. (a) shows the whole fiber and (b) shows a close up of the cladding region. (c) SEM image of a square core silica fiber with a core width of $\sim 150 \mu \mathrm{m}$ (polymer coating not shown). Photograph courtesy of SPI Lasers UK Ltd.

In this paper we show that JAC fibers with non-circular core geometry can be tailor made to a specific core size and NA requirement and that sharply defined vertices in the core shape can be preserved throughout the fiber drawing process. Specifically, we report the fabrication of a JAC fiber with a large square core and show that the near field in this fiber has a top-hat intensity profile without recourse to any externally applied modal mixing, even when the launch is under-filled. As an example demonstration, we show that this fiber can be used to create square ablation marks in an ITO film on a glass substrate.

\section{Fiber design and fabrication}

The square-core JAC fiber reported here was designed specifically for the delivery of a square-shaped beam of intense multi-mode laser light at $1064 \mathrm{~nm}$ for the surface patterning of ITO within an existing ablation system at Exitech Ltd, UK. The target fiber parameters were a core of approximately $400 \mu \mathrm{m}$ in diameter and an NA of about 0.2 to give a suitable beam divergence and image size in the demonstration system. However, we note that the cladding microstructure in this fiber type offers the potential to create much larger values of NA [3, 4]. The effective cladding index in a JAC fiber can be approximated by the modal index of the fundamental mode of the individual silica struts, determined via standard expressions for slab waveguides [3]. Using this assumption, an NA of 0.2 corresponds to a strut width of $\sim 2 \mu \mathrm{m}$. 
Allowing for the solid outer jacket, these requirements imply a fiber diameter (D) of $\sim 0.75$ mm, as illustrated in Fig. 2(a). This is comparable with many commercially available products [1], and corresponds to a minimum long term bend radius $\left(\mathrm{R}_{\min }\right)$ of $\sim 15 \mathrm{~cm}$, which was deemed to be within acceptable bounds for the application considered here. This value of $R_{\min }$ is based on the assumption that there is a linear relationship between $\mathrm{D}$ and the bend radius required to reproduce a certain level of strain and the fact that $R_{\min }=25 \mathrm{~mm}$ for conventional fiber where $\mathrm{D}=125 \mu \mathrm{m}[6]$.

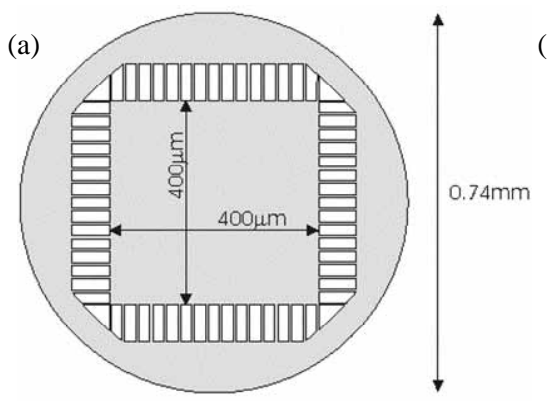

(b)

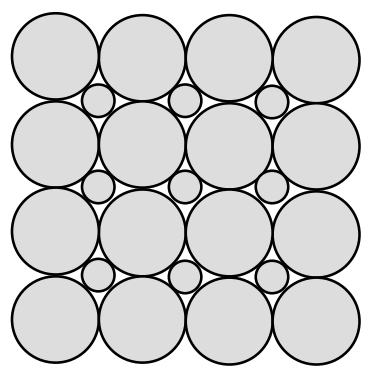

Fig. 2. (a) Schematic drawing of JAC fiber design with $\mathrm{D}=0.74 \mathrm{~mm}$ and a core size of $400 \mathrm{x}$ $400 \mu \mathrm{m}$. (b) Stacking geometry of JAC core

Based on the above design, a JAC fiber with a square core was fabricated using a stack and draw approach. The square core was formed by stacking together many circular silica rods of two different sizes, as illustrated in Fig. 2(b). Note however, that it is not necessary to fabricate this fiber in this way and that similar fibers have also been created with a milled silica rod as the core element. However, the advantage of constructing the core region from stacked elements is that it offers enormous design flexibility in terms of the core shape and composition. A number of thin walled capillaries were stacked around the core and the structure was contained within a jacket tube. Elongated holes surrounding the core region were formed during fiber draw by selectively allowing some pressurized capillaries to expand whilst other freely vented capillaries were allowed to collapse. The spaces between stacked solid core elements were also freely vented during fiber draw so that a solid core was formed. The resulting JAC fiber has a core of width $380 \mu \mathrm{m}$ surrounded by 64 air-holes each approximately 20 by $40 \mu \mathrm{m}$ in dimension, separated by struts $\sim 2 \mu \mathrm{m}$ wide (shown in Fig. 3(a) and $3(b))$. Note that the inner boundary of the microstructure region, which defines the core shape, is finely scalloped. While this effect could be reduced in scale by stacking a larger number of smaller capillaries around the core, it may offer certain advantages in terms of mode mixing, for example.
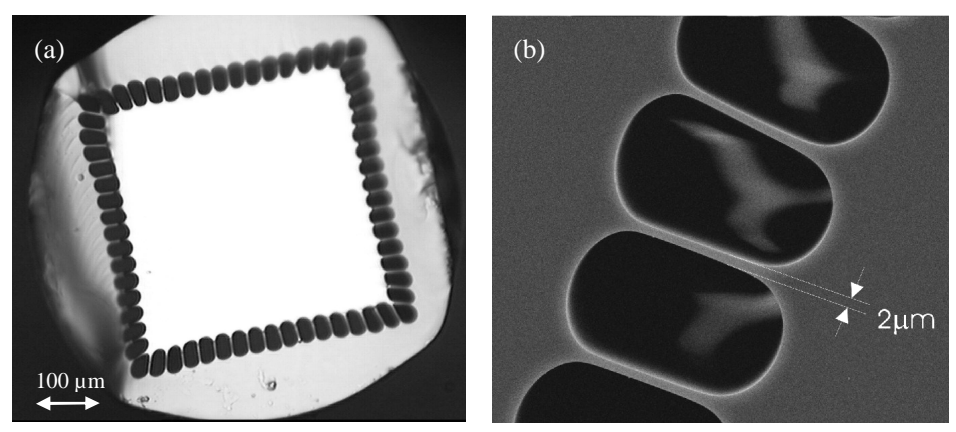

Fig. 3. (a) Optical image of the square-core JAC fiber, (b) SEM image showing the cladding air holes in detail.

$\# 72684$ - \$15.00 USD

(C) 2006 OSA
Received 5 July 2006; revised 24 August 2006; accepted 28 August 2006 30 October 2006 / Vol. 14, No. 22 / OPTICS EXPRESS 10347 


\section{Fiber characteristics}

The fiber output was examined under four different launch conditions; using white light (incandescent bulb) illumination, a single-mode $\mathrm{HeNe}$ laser $(633 \mathrm{~nm}$ ), a single-mode Nd:YAG $(1.06 \mu \mathrm{m})$ laser and a multi-mode laser diode source operating at $915 \mathrm{~nm}$. The intensity profiles of light at the fiber output (imaged onto a CCD camera) are shown in Figs 4(a) - (c) for wavelengths of $633 \mathrm{~nm}, 1.06 \mu \mathrm{m}$ and $915 \mathrm{~nm}$ respectively. The fiber lengths were $3 \mathrm{~m}$, $3 \mathrm{~m}$, and $25 \mathrm{~m}$ respectively. One point to note is that the core shape is reflected in the nearfield profile, with the fine notches at the core edge creating a "postage stamp" effect in the beam shape. We did not observe any noticeable change to the near-field intensity profile in response to bends or twists imposed on the fiber during the course of characterization.
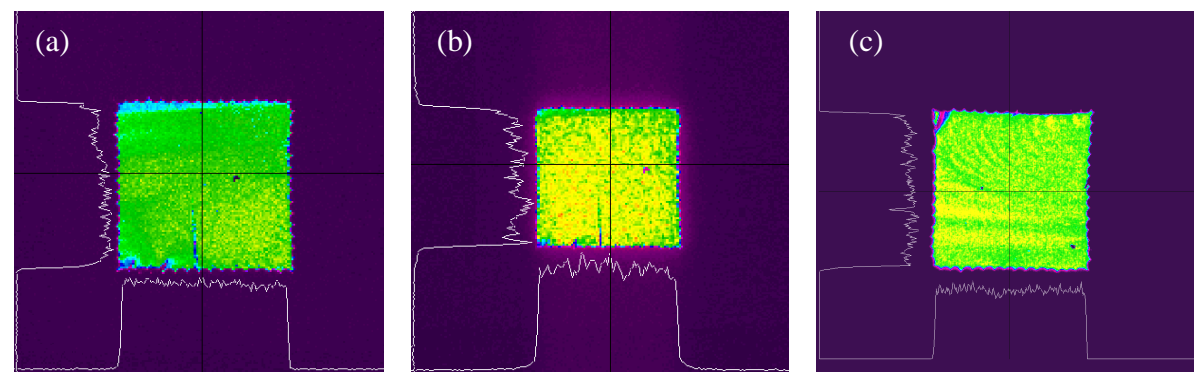

Fig. 4. Near field intensities of the square core fiber at (a) $633 \mathrm{~nm}$, (b) $1.06 \mu \mathrm{m}$ and (c) $915 \mathrm{~nm}$

The far-field output of the fiber was observed to be circular at all wavelengths, with a characteristic red fringe around the perimeter for white light illumination, shown inset in Fig. 5. This demonstrates that the output divergence (i.e. the numerical aperture) increases towards long wavelengths, as expected [3]. However, the numerical aperture (NA) of the light emitted from the fiber output is also found to be dependent on the launch conditions, as illustrated in the following. For the single-mode $633 \mathrm{~nm}$ and $1.06 \mu \mathrm{m}$ sources, light was coupled into approximately $2 \mathrm{~m}$ of fiber using a x 20 microscope objective with an NA of 0.54 . The NA of the fiber in this case was evaluated via measurements of the far-field angle of divergence which is given by NA $=\sin \theta \sim \mathrm{d}_{B} / \mathrm{z}$, where $\mathrm{z}$ is the distance from the fiber end and $\mathrm{d}_{\mathrm{B}}$ is the beam diameter, defined here as the diameter of a circular aperture that transmits $95 \%$ of the total power in the beam. By recording $\mathrm{z}$ as a function of the aperture diameter for $95 \%$ transmission, the NA of fiber was measured to be 0.12 at and 0.11 at $633 \mathrm{~nm}$ and $1.06 \mu \mathrm{m}$ respectively, as shown in Fig. 5. Estimated values of the NA, made using the model in [3], return values of approximately 0.15 and 0.22 at $633 \mathrm{~nm}$ and $1.06 \mu \mathrm{m}$ respectively. These results indicate that the highest-order modes of this multi-mode fiber are not excited by the single-mode launch conditions, which is unsurprising. However, it should be noted that despite this 'under-filled' NA, we find that a good quality square beam shape with a flat intensity profile can still be obtained in short lengths of fiber, as shown previously in Figs 4(a) and (b).

For a multimode launch (using the $915 \mathrm{~nm}$ source and $25 \mathrm{~m}$ of fiber), we find much closer agreement between measured and predicted values of the fiber's NA, which was measured to be $0.21 \pm 0.01$ and is estimated to be 0.20 using the model in [3]. In this case, the NA of the fiber was evaluated from a single width measurement of the unobstructed far-field output projected onto a screen positioned $300 \mathrm{~mm}$ from the fiber exit. This technique was deemed appropriate in this example as several hundred milliwatts of power were available and the edge of the far-field was clearly defined. The agreement between measurement and theory shows that the NA of the fiber is well-filled in this example, indicating that any measurement of transmission or bend loss is representative. The fiber attenuation, measured after cutting back by $23 \mathrm{~m}$ was found to be $\sim 0.06 \mathrm{~dB} / \mathrm{m}$ for this source. To obtain an indication of bend sensitivity, seven coils of radius $8 \mathrm{~cm}$ were introduced in the fiber length. The total bend loss 
induced in this case was measured to be less than $0.1 \mathrm{~dB}$. The near-field output intensity profile for these launch conditions is shown in Fig. 4(c).

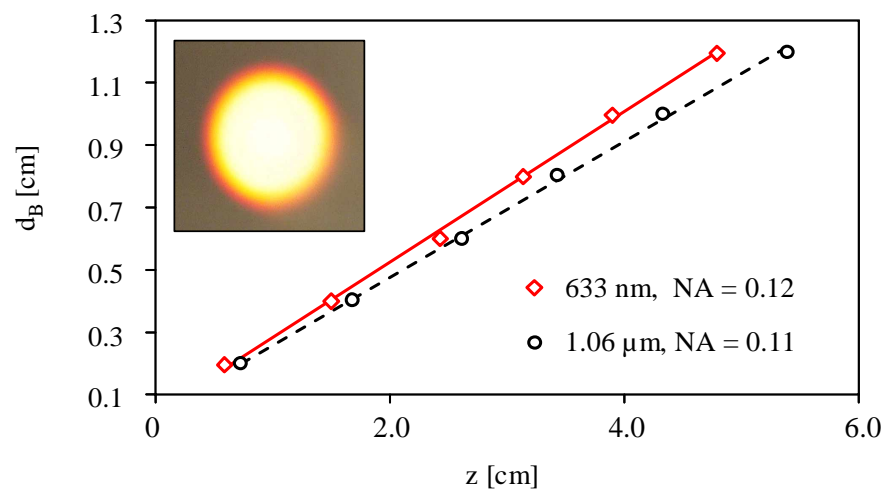

Fig. 5. Beam width $\left(d_{B}\right)$ vs. distance from fiber end $(\mathrm{z})$ for single-mode illumination at $633 \mathrm{~nm}$ and $1.06 \mu \mathrm{m}$. Inset shows far-field output for white light illumination.

\section{Ablation demonstration}

The JAC fiber geometry considered here is specifically designed for the delivery of intense laser radiation from highly multimode sources. Indeed, we have successfully transmitted $35 \mathrm{~ns}$ pulses (rep rate $3 \mathrm{kHz}$ ) with an average power of $\sim 80 \mathrm{~W}$ at $1.06 \mu \mathrm{m}$ from a highly multimode Nd:YAG laser through the circular JAC shown in Fig. 1(a) without incurring damage. The combined coupling and fiber losses in this case were estimated to be $\sim 1 \mathrm{~dB}$. However, with the design of the square JAC fiber pictured in Fig. 3(a) our aim was two-fold; requiring both the capability to transmit sufficiently high powers and also the ability to deliver that power with a square beam profile.

As an example demonstration, the square JAC fiber shown in Fig. 3(a) was used to ablate a thin layer of indium tin oxide (ITO) on a glass substrate, using $30 \mathrm{~ns}$ pulses (rep rate 3.5 $\mathrm{kHz}$ ) with $6.1 \mathrm{~W}$ average power at $1.06 \mu \mathrm{m}$ from a highly multi-mode Q-switched Nd:YAG laser. The coupling efficiency between the laser and the fiber was $\sim 77 \%$. However, we note it should almost certainly be possible to improve this by more appropriate lens selection and fiber end face preparation. The system used in these ablation trials is shown in Fig. 6(a). The size of the image at the ITO surface could be altered by adjusting the distance between the fiber exit and the imaging lens $\left(\mathrm{d}_{\mathrm{L}}\right)$ and the image was brought into focus by adjusting the lens to work piece distance. During ablation, the work piece was translated horizontally at a speed of $0.5 \mathrm{~ms}^{-1}$ to spatially separate each pulse of the laser at the ITO surface. In this way, each pulse of the laser ablated a single square in the ITO film. An example line of ablated features, each $\sim 115 \mu \mathrm{m}$ in width, is shown in Fig. 6(b). In this example, $d_{L}=50 \mathrm{~mm}$ and the average power at the ITO surface was measured to be $3.0 \mathrm{~W}$, corresponding to a fluence of $6.5 \mathrm{Jcm}^{-2}$ and a throughput efficiency of $\sim 50 \%$, which is already comparable to a commercial system. By adjusting these parameters, features of similar quality were also produced ranging in size from $70-120 \mu \mathrm{m}$. It should be appreciated that the fine notches at the edge of the fiber, which result in a 'postage stamp' effect in the near-field, as shown in Figs 3 and 4, are not apparent in the ablated features. We note that for a fixed feature size there is scope to improve the overall throughput efficiency by tailoring both the fiber parameters and the lens system.

Although the fine notches visible in the outer edge of the fiber near-field are smoothed out in the ablated profile, other imperfections are present, particularly along the top and left hand sides of each feature. For the trials reported here the fiber was cleaved by hand using a small ceramic fiber tile. This method of end-face preparation is often applied, with good results, to a wide variety of microstructured fiber designs where conventional mechanical cleavers are not suitable. However, the geometry of JAC fibers permits the propagation of conchoidal 
fractures within the fiber core and inspection of the fiber end showed poor surface flatness within this region. Indeed, the concentric circular ripples typical of this classic glassy fracture are evident in the top half of the near-field intensity profile shown in Fig. 4(c). This intensity profile also shows an additional distortion in the top left corner, where a small section of the fiber core has been chipped away. The surface imperfections visible in Fig. 4(c) are typical for this particular fiber type and correlate well with the imperfections in the ablated features shown in Fig. 6(b), indicating that improving the definition of the ablated features is largely a matter of improving the fiber end-face quality. This is relatively trivial and there are a variety of techniques that can be used to produce high quality end-faces in microstructured fibers. For example, $\mathrm{CO}_{2}$ laser cleaving, which has been shown to produce excellent results in conventional solid fibers, has been successfully applied to microstructured fibers [6].

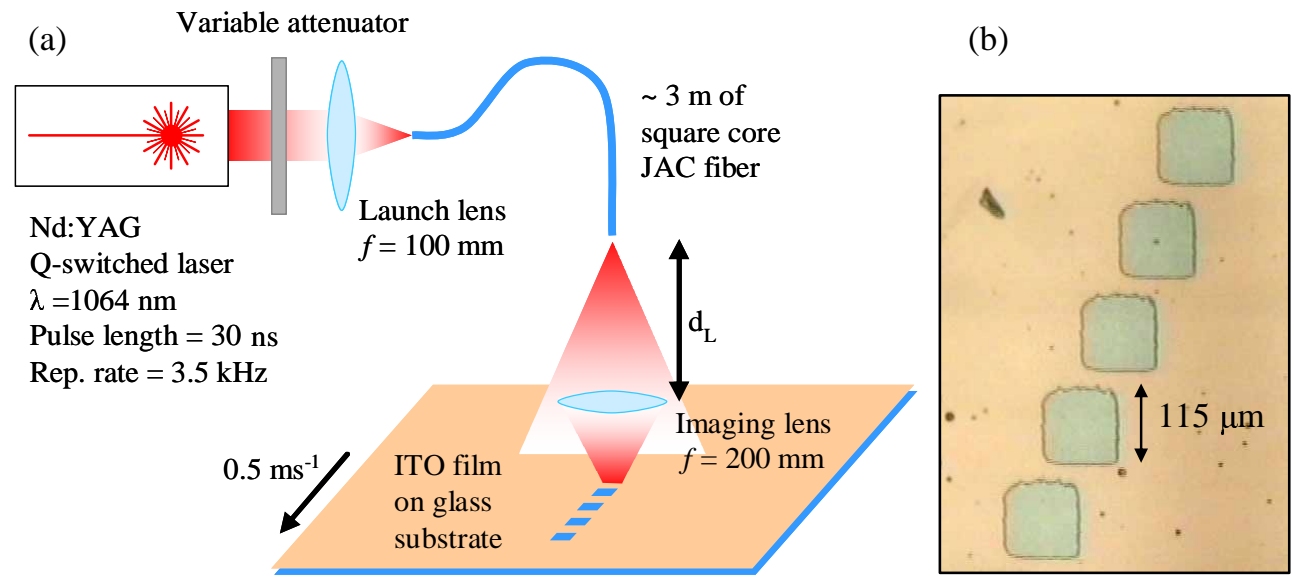

(b)

Fig. 6. (a) Schematic of the system used in ablation trials. (b) Features ablated in an ITO film using the system illustrated in (a) with $d_{L}=50 \mathrm{~mm}$. Average power at fiber input and ITO surface $=6.1$ and $3.0 \mathrm{~W}$ respectively, corresponding to a fluence of $6.5 \mathrm{Jcm}^{-2}$ at the ITO surface.

\section{Discussion and conclusions}

A jacketed air-clad fiber with a square shaped core has been successfully fabricated using microstructured fiber technology. As an example demonstration, we have shown that this type of fiber offers a practical way of producing square shaped ablation marks in thin layers of indium tin oxide (ITO) without the need for complex beam shaping optics. We note that this patterning process can be simply adapted to a wide range of source wavelengths (limited only by the transmission properties of silica glass) and is also applicable to resin based layers, such as those used in the production of liquid crystal displays (LCD). The simplicity of this solution offers significant advantages in terms of power conservation and ease of use for high power applications requiring non-circular beam shapes. Furthermore, although this process has been demonstrated using a large solid state laser, one could easily envisage the creation of a compact system by using a fiber laser source.

\section{Acknowledgments}

The authors acknowledge the support of an OSDA DTI Link grant (PFIDEL), EPSRC grant number: GR/SO5793-01 and to Andy Piper and Michael J. Lovelady of SPI Lasers plc for their assistance with the measurements at $915 \mathrm{~nm}$. 